\title{
La actitud del estudiantado, clave para entender la mejora de sus resultados académicos en un entorno de ICSSHGFOWRRP
}

Ruiz Jiménez, M.Carmen'; Martínez Jiménez, Rocío ${ }^{a}$; Licerán Gutiérrez, Ana ${ }^{\text {; }}$; García Marti, Eliaa ; Moreno Fuentes, Elena ${ }^{c}$ y Risueño Martínez, Jesús Juanc ${ }^{c}$.

${ }^{a}$ Departamento de Organización de Empresas, Marketing y Sociología de la Universidad de Jaén. ${ }^{b}$ Departamento de Economía Financiera y Contabilidad de la Universidad de Jaén. ${ }^{c}$ Departamento de Didáctica de la Lengua Inglesa del Centro Universitario Sagrada Familia de Úbeda. Correos electrónicos: cruiz@ujaen.es; rmartine@ujaen.es; aliceran@ujaen.es; egarcia@ujaen.es; emoreno@fundacionsafa.es; jrisueno@fundacionsafa.es

\section{\$EWWDFW}

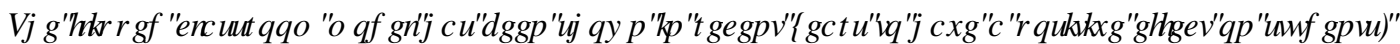

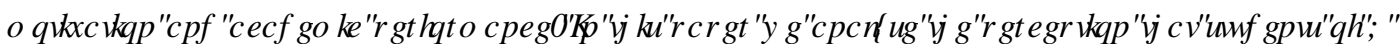

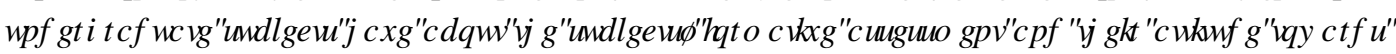

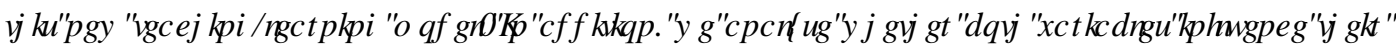

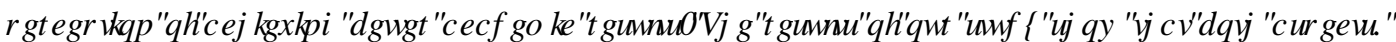

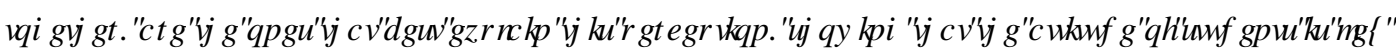

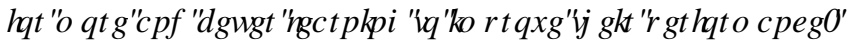

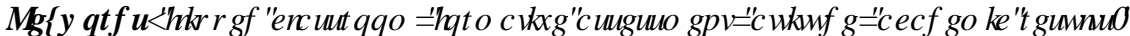

\begin{abstract}
5 HXP HQ

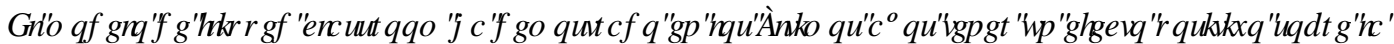

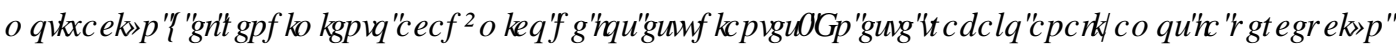

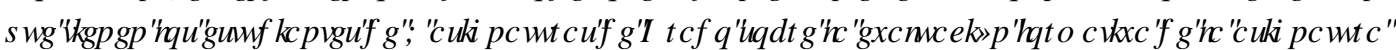

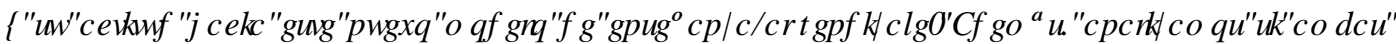

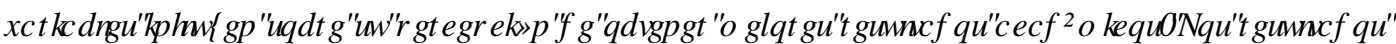

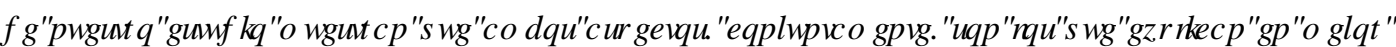

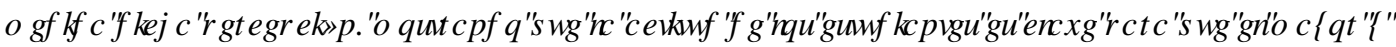

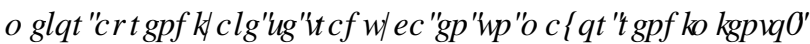

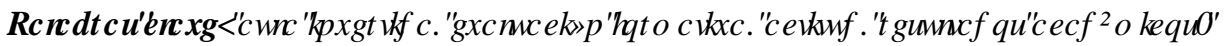

\section{Introducción}

El principal objetivo del sistema educativo ha de ser garantizar a los estudiantes el logro de una adecuada formación, que incluya conocimientos y habilidades, que les faciliten su acceso al mercado laboral en las condiciones que las empresas demandan. Este logro está garantizado si se aplican metodologías donde el estudiante se enfrente a la resolución de situaciones complejas y reales, donde tenga que aprender a tomar decisiones ante determinados problemas y donde tenga que aprender a pensar por sí mismo con una actitud crítica y analítica. Estas situaciones apuntan hacia metodologías más activas en las cuales se traslade a los estudiantes la responsabilidad de su propio aprendizaje. En este marco, la propuesta que ha mostrado un mayor potencial para incorporar un aprendizaje activo en el aula es el modelo de aula invertida (Prober y Heath, 2012). 
El origen del modelo de aula invertida o ICSSHGFCDWRRP lo encontramos en el año 2006 en los profesores de la Escuela Secundaria de Denver en Colorado, Jon Bergmann y Aaron Sams. Aunque inicialmente la base era sustituir una explicación en el aula por un vídeo, especialmente dirigido a aquellos estudiantes que no podían asistir a clase, el concepto ha evolucionado hacia una metodología más amplia y en continuo desarrollo que se va nutriendo de una cada vez más extensa evidencia académica y científica.

El aula invertida cambia el contexto y la forma de trabajar tanto del profesor como de los estudiantes, dentro y fuera del aula. En el modelo tradicional, los estudiantes sólo tienen que "sentarse y recibir contenidos"; esperan que el profesor les explique qué deben aprender, cómo aprenderlo, cuándo hacerlo y cómo demostrar después que lo han aprendido. El aula invertida, sin embargo, desplaza al docente del centro del proceso educativo y cede la responsabilidad de su aprendizaje al estudiantado. Así, el docente facilita a los estudiantes, previamente a la sesión de clase, la información (audiovisual o escrita) para que éstos la analicen, estudien y comprendan los contenidos de manera autónoma. Los alumnos estudian de forma individual y se preparan para la clase. De este modo, se libera tiempo en el aula que se puede dedicar a resolver dudas, a discutir trabajando en equipo y de forma colaborativa o a aplicar y reforzar lo aprendido, con el potencial uso de distintas herramientas digitales. Así, se crean entornos de aprendizaje centrados en el alumno, como el aprendizaje basado en la indagación, el estudio de casos, la resolución de problemas, el aprendizaje basado en proyectos y la evaluación formativa (Prieto y Giménez, 2020a).

El aula invertida se basa en un aprendizaje personalizado puesto que ofrece la posibilidad de que cada estudiante aprenda a su ritmo, atendiendo así a la diversidad de capacidades que encontramos en el aula. Se basa en una metodología inductiva, que es más eficaz que la metodología deductiva tradicional. Y, lo más importante de todo, el resultado de todo el proceso ya no debe ser que "se explique muy bien la materia" sino que "el estudiante aprenda la materia".

Existe una amplia literatura de los efectos del aula invertida sobre múltiples elementos del proceso educativo. En el caso de los resultados académicos, el aula invertida conlleva mejorar resultados en la evaluación si está unida al aprendizaje activo (Jensen et al., 2015). La utilización del método de aula invertida amplía el espacio de trabajo, permite una distribución diferente de las dinámicas del aula antes, durante y después de clase, ofreciendo a los estudiantes la oportunidad de interactuar antes y de manera más sólida con los materiales formativos, lo que conlleva una mejor lectura que se ve reforzada con las actividades de clase y que implica mejores resultados académicos (Prieto et al., 2021; Strelan et al., 2020). Las metodologías basadas en la capacidad del estudiante de controlar y decidir sobre sus tiempos de trabajo han permitido un mayor impacto educativo (Delgado et al., 2018).

Junto a ello, un aspecto sobre el que se percibe un mayor común acuerdo es que con la aplicación de estrategias activas de enseñanza-aprendizaje, el profesorado ha conseguido un cambio en la actitud del estudiantado hacia la enseñanza. Este cambio de actitud se puede apreciar en aspectos como la asistencia a clase, la participación, el compromiso, la implicación o la motivación. Como muestran diversos estudios, el aula invertida incrementa el compromiso (preparación y atención), mejora la actitud y favorece la implicación en el proceso de aprendizaje y el desarrollo de habilidades y competencias de los estudiantes (White et al., 2017; Murillo-Zamorano et al., 2019; Zheng et al., 2020).

Otro elemento importante sobre el que pivota el modelo de aula invertida, como indican Flores et al. (2016) es la evaluación. La capacidad organizativa del estudiante ya ha demostrado unos mejores resultados de aprendizaje y, para ello, la evaluación asociada al proceso es clave (Meusen-Beekman et al., 2015). El aula invertida es idónea para la evaluación de los resultados de aprendizaje de los estudiantes porque se puede trabajar con evaluación continua a la vez que ofrecer mejor feedback a través de la evaluación formativa. Esta última implica un proceso de retroalimentación entre todos los agentes educativos, sin asociarse a una calificación y con el objetivo de mejorar tanto el aprendizaje de los estudiantes como la propia práctica 
docente (Hortigüela et al., 2019). Así, la evaluación formativa permite al estudiante ser más consciente de lo que aprende y le ofrece la posibilidad de autorregular su aprendizaje. Y, combinada con el uso de metodologías activas y participativas con las cuales se permite a cada estudiante valorar tanto su trabajo como el de los demás, se favorece el empleo de diferentes canales de retroalimentación: orales, escritos, en video, individuales, grupales...

Los efectos del aula invertida sobre todos los elementos del proceso educativo indicados en los párrafos anteriores se traducen en un mejor aprendizaje, más significativo, con una mejor comprensión y retención de lo aprendido (Prieto et al. 2021). Sin embargo, según Goodwin y Miller (2013) la evidencia sobre el modelo invertido está aún por llegar, reconociendo que aún no existe una gran base de investigación científica que indique exactamente la eficacia de las aulas que siguen dicho modelo. En esta línea planteamos este trabajo, buscando aportar más evidencia sobre los efectos del aula invertida desde la perspectiva de los estudiantes universitarios, intentando conocer si existe y cómo es la relación entre las dinámicas aplicadas y sus resultados.

\section{Objetivos}

En todo proceso de innovación educativa es importante conocer el impacto que están teniendo en los estudiantes los cambios aplicados en el proceso de enseñanza-aprendizaje, así como identificar su percepción sobre su nivel de implicación y participación en la clase y el nivel de consecución de los resultados de aprendizaje. Para ello, es necesario planificar cómo se va a evaluar el efecto de la innovación sobre aquellos aspectos que más interesan. En este caso, nos centramos en analizar la percepción de los estudiantes sobre su grado de satisfacción, su actitud ante la nueva dinámica, dentro y fuera del aula, y la valoración sobre su grado de aprendizaje y resultados esperados.

Por todo lo anterior, el presente trabajo se plantea como principal objetivo mostrar los resultados obtenidos con la experiencia de aula invertida en distintas asignaturas relacionadas con el ámbito de la gestión de empresas, impartidas en diferentes Grados en la Universidad de Jaén, y relacionadas con la educación infantil y primaria, impartidas en Grados en las Escuelas Profesionales Sagrada Familia (SAFA) de Úbeda (Jaén).

Más concretamente, podemos definir como objetivos específicos profundizar en la percepción que tienen los estudiantes sobre la experiencia de aula invertida para:

- Conocer si esta experiencia, a través de la evaluación formativa, les ha ayudado a conseguir un aprendizaje más significativo, entendiendo como tal comprender y aprender más y mejor el contenido de las materias.

- Conocer si este sistema de trabajo les ha motivado, gustado y divertido, lo que creemos que es un reflejo de una actitud positiva hacia esta nueva metodología.

- Conocer sus expectativas sobre los resultados académicos que alcanzarán.

Junto a ello, creemos que es posible testar la existencia de una relación entre las variables mencionadas anteriormente, de manera que su percepción sobre los aspectos de la evaluación formativa y su actitud hacia la experiencia puedan explicar la percepción sobre su rendimiento académico. 


\section{Desarrollo de la innovación}

Tanto el desarrollo de esta experiencia innovadora en el aula universitaria como la difusión de sus resultados, ha sido posible gracias a la concesión del proyecto de Innovación Docente titulado "Aulas invertidas (Flipped Classroom): Una nueva forma de enseñar y aprender", concedido por la Universidad de Jaén en el marco de su "Plan de Innovación y Mejora Docente (PIMED-UJA 2019).

El modelo de aula invertida se ha puesto en marcha por parte de seis profesores de la Universidad de Jaén, en distintas asignaturas de grado de la Facultad de Ciencias Sociales y Jurídicas y del Centro Universitario Sagrada Familia de Úbeda, durante el primer cuatrimestre del curso 2020-2021. La mayoría de los profesores veníamos aplicando aula invertida en cursos anteriores; sin embargo, debido a la situación de pandemia hemos tenido que adaptar a modalidad online algunas de las dinámicas que veníamos utilizando en modo presencial puesto que la docencia durante el cuatrimestre ha sido híbrida o alterna (mitad de grupo online y mitad presencial).

$\mathrm{Al}$ comienzo del cuatrimestre cada profesor explicó a sus estudiantes qué es la docencia invertida y cuál/les sería/n las dinámicas a seguir tanto en la sesión de clase como antes de clase. Se han utilizado distintas metodologías activas que se recogen en la siguiente tabla, combinadas con distintos procedimientos y herramientas de evaluación. En este sentido, les explicamos a los estudiantes que la evaluación sería formativa y sumativa, además de continua y final. En la siguiente tabla se recogen las distintas dinámicas activas que se han aplicado así como el procedimiento de evaluación, para cada una de las asignaturas.

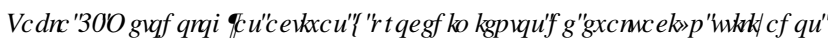

\begin{tabular}{|c|c|c|c|c|c|}
\hline \multirow[t]{2}{*}{ Grado } & \multirow[t]{2}{*}{ Asignatura } & \multirow[t]{2}{*}{ Metodologías activas } & \multicolumn{3}{|c|}{$\begin{array}{l}\text { Procedimiento de } \\
\text { evaluación }\end{array}$} \\
\hline & & & $\mathrm{EF}$ & ES & $\mathrm{EC}$ \\
\hline $\begin{array}{l}\text { Administración y } \\
\text { Dirección de } \\
\text { Empresas y Derecho }\end{array}$ & Dirección estratégica I & Videos y estudio de casos & SI & SI & SI \\
\hline Educación infantil & $\begin{array}{l}\text { Lengua extranjera y su } \\
\text { didáctica (inglés) }\end{array}$ & $\begin{array}{l}\text { Videos (Edpuzzle), audios y lecturas } \\
\text { previas. Gamificación (Kahoot, } \\
\text { Quizizz, mentimeter, Break-out } \\
\text { educativo). Lluvia de ideas, debates. }\end{array}$ & SI & SI & SI \\
\hline Educación primaria & $\begin{array}{l}\text { Aprendizaje y Enseñanza } \\
\text { de Lenguas Extranjeras } \\
\text { (Inglés) }\end{array}$ & $\begin{array}{l}\text { Videos (Edpuzzle), audios y lecturas } \\
\text { previas. Gamificación (Kahoot, } \\
\text { Quizizz, mentimeter, Break-out } \\
\text { educativo). Lluvia de ideas, debates. }\end{array}$ & SI & SI & SI \\
\hline Educación Primaria & $\begin{array}{l}\text { Metodología y recursos } \\
\text { para la enseñanza de } \\
\text { lenguas } \\
\text { (inglés) }\end{array}$ & $\begin{array}{l}\text { Videos (Edpuzzle), audios y lecturas } \\
\text { previas. Gamificación (Kahoot, } \\
\text { Quizizz, mentimeter. Lluvia de ideas, } \\
\text { debates. }\end{array}$ & SI & SI & SI \\
\hline
\end{tabular}




\begin{tabular}{|c|c|c|c|c|c|}
\hline Estadística y Empresa & $\begin{array}{l}\text { Administración } \\
\text { empresas }\end{array}$ & $\begin{array}{l}\text { JITeaching, gamificación (Kahoot, } \\
\text { Quizizz, Mentimeter), indagación, } \\
\text { estudio de casos }\end{array}$ & SI & SI & SI \\
\hline $\begin{array}{l}\text { Finanzas y } \\
\text { Contabilidad }\end{array}$ & Contabilidad de gestión II & 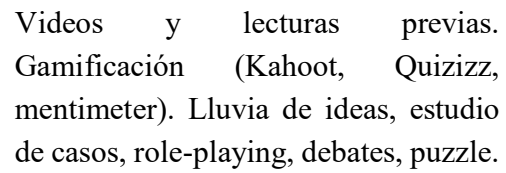 & SI & SI & SI \\
\hline $\begin{array}{l}\text { Finanzas y } \\
\text { Contabilidad }\end{array}$ & $\begin{array}{l}\text { Gestión de recursos } \\
\text { humanos }\end{array}$ & $\begin{array}{l}\text { Lecturas previas. Gamificación } \\
\text { (Kahoot, Socrative). Lluvia de ideas, } \\
\text { estudio de casos, role-playing, debates. }\end{array}$ & SI & SI & SI \\
\hline $\begin{array}{l}\text { Ingeniería } \\
\text { Organización } \\
\text { Industrial }\end{array}$ & $\begin{array}{l}\text { Organización del trabajo y } \\
\text { factor humano }\end{array}$ & $\begin{array}{l}\text { Lecturas previas. Gamificación } \\
\text { (Kahoot). Lluvia de ideas, estudio de } \\
\text { casos, role-playing, debates. }\end{array}$ & SI & SI & SI \\
\hline Relaciones Laborales & $\begin{array}{l}\text { Organización } \\
\text { administración } \\
\text { empresas }\end{array}$ & $\begin{array}{l}\text { JITeaching, gamificación (Kahoot, } \\
\text { Quizizz, Mentimeter), indagación, } \\
\text { estudio de casos }\end{array}$ & SI & SI & SI \\
\hline
\end{tabular}

EF: Evaluación Formativa $\quad$ ES: Evaluación Sumativa $\quad$ EC: Evaluación Continua. Fuente: Elaboración propia.

Con objeto de conocer qué percepción tenían nuestros estudiantes respecto a la aplicación de la docencia invertida en las asignaturas, los últimos días de clase del primer cuatrimestre les pedimos que completaran un cuestionario voluntario y anónimo que constaba de 15 preguntas de valoración sobre distintos aspectos (motivación, comprensión, autonomía, eficacia en el aprendizaje...), usando una escala Likert de 1 a 5 (totalmente en desacuerdo - totalmente de acuerdo). Además, les planteamos una pregunta abierta sobre valoración general del aula invertida así como aspectos positivos/negativos y propuestas de mejora. El número total de estudiantes matriculados en las nueve asignaturas es de 270 y respondieron este cuestionario un total de 146 estudiantes, lo que supone una tasa de respuesta del 54,07\%. Por asignaturas, podemos ver el porcentaje de respuesta a este cuestionario en la siguiente tabla.

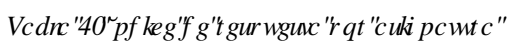

\begin{tabular}{lccc}
\hline Asignatura & $\begin{array}{c}\mathbf{N}^{\mathbf{0}} \text { estudiantes } \\
\text { matriculado }\end{array}$ & $\begin{array}{c}\mathbf{N}^{\mathbf{0}} \text { cuestionarios } \\
\text { recibidos }\end{array}$ & $\begin{array}{c}\text { Tasa de } \\
\text { respuesta }\end{array}$ \\
\hline Administración de empresas & 26 & 10 & $38.46 \%$ \\
$\begin{array}{l}\text { Aprendizaje y enseñanza de las lenguas } \\
\text { extranjeras }\end{array}$ & 38 & 17 & $44,74 \%$ \\
$\begin{array}{l}\text { Contabilidad de Gestión II } \\
\text { n }\end{array}$ & 48 & 32 & $66,67 \%$ \\
\hline
\end{tabular}


Dirección Estratégica I

Gestión de RRHH

Lengua extranjera y su didáctica

Metodología y recursos para la enseñanza de lenguas extranjeras

Organización del trabajo y factor humano

Organización y administración de empresas

TOTAL
19

15

33

27

27

37
4

12

2

20

26

23

146
$74,07 \%$

$96,30 \%$

$62.16 \%$

$21,05 \%$

$80,00 \%$

$6,06 \%$

$54,07 \%$

Fuente: Elaboración propia.

Posteriormente, procedimos al análisis de los resultados de dicho cuestionario mediante técnicas estadísticas para obtener conclusiones de cómo ha afectado la implantación de este método al proceso de aprendizaje del alumnado. En concreto, como comentamos en los objetivos, analizamos la percepción que los estudiantes tienen sobre la evaluación formativa y su actitud ante la nueva metodología, así como su percepción de los resultados que obtendrán en la asignatura. Además, testamos un modelo que analiza la relación entre la evaluación formativa y la actitud del estudiante con la percepción de los resultados en la evaluación de dichos estudiantes.

Las variables que se pretenden medir son variables no observables directamente, pues se refieren a aspectos del comportamiento y percepciones. Para este tipo de análisis, las técnicas tradicionales de estimación no son suficientes, al no poderse obtener una expresión empírica clara de las variables (Rigdon, 2012). En este sentido, han emergido en la actualidad técnicas estadísticas como los Modelos de Ecuaciones Estructurales (Structural Equation Models, SEM), capaces de incorporar explícitamente esa noción psicométrica a las variables no observables a su perspectiva econométrica (Fornell y Larcker, 1981; Roldán y Sánchez-Franco, 2012). Así, las variables no observables directamente se pueden inferir mediante una serie de indicadores que sean representativos de ese concepto (Henri, 2007; Ulman, 2006). En nuestro estudio, utilizaremos como indicadores las respuestas a determinados ítems del cuestionario. Una representación esquemática del modelo a estimar mediante SEM (en particular, utilizando la técnica de Partial Least Squares -PLS-) se muestra en la Figura 1. 


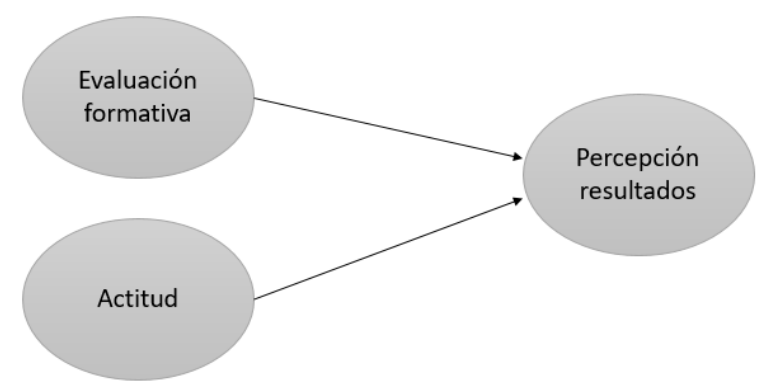

) $L \amalg \amalg \Pi 0$ RGHAIGHDQ QUV

Las tres variables analizadas, con sus correspondientes indicadores, son:

- $\quad$ ( 9 \$ 8 \$\&, I 1 1 ) 250 \$7,9\$(variable independiente): refleja la percepción del estudiantado sobre los beneficios de la metodología de aula invertida de cara a la preparación y desarrollo de la asignatura a lo largo del curso. Para ello, se seleccionan los ítems "Esta metodología me ha permitido comprender mejor la teoría", "Aprendí más y mejor con este método", "Las actividades realizadas me permiten aprender el material de estudio más eficazmente”, y "Esta metodología me ha permitido trabajar la materia de forma continua y no dejar todo para antes del examen".

- $\quad \$ \& 7,78^{\prime} \square($ variable independiente): refleja la percepción del estudiantado sobre la capacidad motivacional del aprendizaje basado en aula invertida. Específicamente, se utilizan los siguientes ítems: "Este método me ha motivado más que una clase tradicional", "Me he divertido en el proceso de aprendizaje", y "Me ha gustado la metodología de aula invertida seguida en clase".

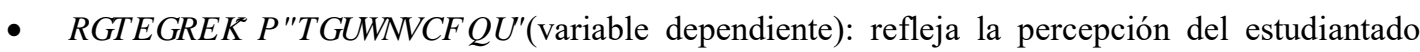
sobre cómo la metodología de aula invertida repercute en los resultados que obtendrá en la evaluación de la asignatura. En concreto, se utilizan los siguientes ítems: "He podido tener una autoevaluación de mi proceso de aprendizaje” y "Creo que esta metodología más activa me va a permitir mejorar mis calificaciones".

En la siguiente sección presentamos los resultados obtenidos del análisis descriptivo de los ítems seleccionados así como de la regresión mediante PLS aplicada al modelo descrito en la Figura 1.

\section{Resultados}

Siguiendo la metodología de PLS, presentamos los resultados obtenidos sobre la validez de la medición de las variables, así como del poder explicativo de la relación entre dichas variables.

En primer lugar, en cuanto a las variables se refiere, todas ellas muestran niveles aceptables de fiabilidad. En particular, los resultados (disponibles a petición de cualquier persona interesada), muestran que todos los ítems son válidos para medir el concepto al que se asocian, siendo representativos de éste; esto se demuestra por el hecho de que todos los indicadores (ítems del cuestionario) asociados a sus respectivas variables tienen una carga factorial superior a 0.7 (Carmines y Zeller, 1979). Asimismo, estos indicadores no sólo son representativos de las variables, sino que también de manera conjunta (agrupados como se ha descrito anteriormente), explican correctamente las variables; esta capacidad de los indicadores de explicar de manera agregada el concepto que representan, conocido como fiabilidad de consistencia interna (Mackenzie et al., 2011; Roldán y Sánchez-Franco, 2012) se observa por el hecho de que las variables en 


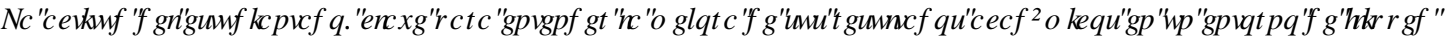 \\ FOWWRRP}

conjunto muestran valores para el alpha de Cronbach y para el índice de fiabilidad compuesta superiores, ambos, a 0.7 (Mackenzie et al., 2011). Por último, todos los indicadores explican una buena proporción del comportamiento (variación) de la variable a la que se asocian, como muestran los valores de la varianza media (Average Variance Extracted, AVE) superiores a 0.5 (Roldán y Sánchez-Franco, 2012).

Comprobada la validez de las medidas, creemos que es interesante conocer en primer lugar la valoración que los estudiantes dan a los distintos ítems analizados. En la tabla 3 mostramos los resultados del análisis descriptivo de las preguntas realizadas en el cuestionario, con la media y la desviación típica en cada caso. Como podemos comprobar, la valoración media es muy satisfactoria en todas las variables (por encima de 3.9 en todas ellas). Esto es una muestra de que los estudiantes perciben mucho más útil para su rendimiento académico esta metodología de aula invertida en comparación con la metodología tradicional.

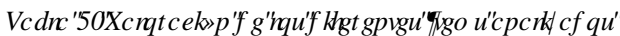

\begin{tabular}{lc}
\hline VARIABLE $\quad$ Item & Media $\begin{array}{l}\text { Desviación } \\
\text { típica }\end{array}$
\end{tabular}

EVALUACIÓN FORMATIVA

ACTITUD

PERCEPCIÓN DE RESULTADOS

Esta metodología me ha permitido comprender mejor la 4.08
teoría

1.03

Aprendí más y mejor con este método

Las actividades realizadas me permiten aprender el material

4.14

1.06 de estudio más eficazmente

Esta metodología me ha permitido trabajar la materia de

4.14

1.01 forma continua y no dejar todo para antes del examen

Este método me ha motivado más que una clase tradicional

3.97

Me he divertido en el proceso de aprendizaje

Me ha gustado la metodología de aula invertida seguida en

4.10 clase

He podido tener una autoevaluación de mi proceso de 4.02 1.01 aprendizaje

Creo que esta metodología más activa me va a permitir 3.99 0.96 mejorar mis calificaciones

A continuación, centramos nuestra atención en la relación entre las variables del modelo. Para ello, presentamos en la Tabla 4 los resultados de la magnitud de la relación entre las variables independientes y dependiente (betas), con su significación estadística (p-valor), así como el poder explicativo del modelo (R2 ajustado). 


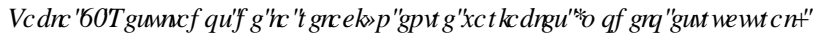

\begin{tabular}{lcccc}
\hline & R2 Ajust & Beta & Estad. T & P-Valores \\
\hline ACTITUD & & $0.438^{* * *}$ & 3.401 & 0.001 \\
EVALUACIÓN_FORMATIVA & 0.548 & & & \\
& & $0.344^{* * *}$ & 2.589 & 0.01 \\
\hline
\end{tabular}

Podemos observar que las variables explicativas tienen un poder de estimación apropiado, como muestra un $\mathrm{R}^{2}$ ya ajustado de 0.548 . Además, ambas variables son estadísticamente significativas, incluso al $99 \%$, con un efecto sobre la variable dependiente de magnitud alta, tal como muestran los coeficientes beta de ambas variables. El modelo mejoraba tanto $\mathrm{su}^{2}$ ajustado como los p-valores al incluir simultáneamente ambas variables independientes, lo que demuestra que la conjunción de ambas es lo que realmente explica la percepción de resultados de los estudiantes. Por último, comprobamos que la relación entre las variables independientes y la dependiente es, en ambos casos, de signo positivo.

Todo lo anterior se traduce en que la percepción de los estudiantes de obtener mejores resultados en la evaluación final de la asignatura depende positivamente tanto de la actitud con la que el estudiante afronte su aprendizaje mediante estas metodologías activas como de la evaluación formativa que retroalimenta dicho proceso y que les ayuda a trabajar mejor durante todo el curso.

\section{Conclusiones}

El modelo de aula invertida nos ofrece la oportunidad de experimentar con otros sistemas de enseñanza y aprendizaje distintos del modelo tradicional. El objetivo de este trabajo ha sido conocer la percepción que tienen los estudiantes respecto a la aplicación de la docencia invertida en nueve asignaturas de distintos Grados de la Universidad de Jaén, durante el primer cuatrimestre del curso 2020-21. Aunque hay un abanico de temas relacionados con los efectos del aula invertida sobre el proceso de enseñanza-aprendizaje, en este caso hemos puesto la atención en los siguientes aspectos: saber cómo las dinámicas de aula invertida modelan la actitud de los estudiantes en su rutina de clase, conocer su valoración sobre el trabajo continuado y la evaluación formativa y conocer su percepción sobre los resultados esperados.

Para conseguir nuestro objetivo, hemos realizado una encuesta a nuestros estudiantes en la que queremos conocer su percepción sobre diversos ítems, incluidos, más concretamente, en las siguientes tres variables: actitud, evaluación formativa y percepción de resultados. En este sentido, uno de los primeros pasos a dar fue comprobar la fiabilidad de los ítems empleados como indicadores de cada una de las variables. Ello se realizó mediante un análisis estadístico con PLS, verificándose en todos los casos la validez y fiabilidad de los mismos.

Por otra parte, del análisis estadístico (descriptivo y de regresión) realizado, podemos confirmar que los resultados están en línea con la doctrina en literatura sobre los beneficios del aula invertida.

Así, en primer lugar, en relación a la actitud, hemos comprobado cómo la aplicación del modelo de aula invertida ha supuesto un cambio en la actitud de nuestros estudiantes. Tal y como lo han manifestado en sus respuestas, se han sentido más motivados y se han divertido y lo han disfrutado más que si lo comparamos con el sistema tradicional. Esto nos permite afirmar que cuando se cede al alumno un papel 
más activo en el proceso de aprendizaje, se siente protagonista, se implica y se motiva más. A estos resultados también contribuye el hecho de que el aula invertida permite trabajar en entornos tecnológicos con los que se sienten muy identificados. Como recogen Prieto et al., (2021), el efecto del aula invertida sobre el aprendizaje se basa en su capacidad para estimular a los alumnos a hacer cosas para aprender e implicarse más en su propio aprendizaje. En la misma línea se encuentran los trabajos de Danker (2015), Flores et al. (2016) y Chih-Yuan y Wu (2016), que evidencian que apostar por metodologías como el aula invertida hace que los estudiantes muestren una actitud positiva hacia las materias de estudio.

Sobre el proceso de evaluación, la docencia invertida apuesta por una evaluación continuada durante todo el curso con objeto de conseguir una evaluación que se entrelace con el proceso de aprendizaje y que promueva la generación de mejores preguntas y del aprendizaje a partir de los errores (Tourón y Santiago, 2015). Como indican Salas y Vicente (2020), para conseguir buenos resultados, debemos hacer un reparto equitativo de tareas y actividades de forma que los estudiantes trabajen de manera continuada y que asuman la responsabilidad de su propio aprendizaje durante todo el curso. Nuestros estudiantes han manifestado que el trabajo continuado les ha permitido mantener un ritmo de aprendizaje constante. Gracias a esto, y a la posibilidad de recibir retroalimentación mediante la evaluación formativa, han logrado asimilar mejor los contenidos y aprender más. Del mismo modo, y en línea con lo anterior, han manifestado tener mejores perspectivas sobre los resultados de la evaluación final de las asignaturas. Los resultados así confirman cómo el esfuerzo que hace el estudiante en la preparación previa de las clases, el trabajo contínuo y el feedback que recibe, son elementos importantes que éste valora y que se traducen en una mejor asimilación de contenido, lo cual también influye en una mejor percepción de sus resultados.

A modo de conclusión final, podemos decir que, como nuestro modelo ha puesto de manifiesto, la percepción de los estudiantes de obtener mejores resultados en la evaluación final de la asignatura depende positivamente tanto de la actitud con la que el estudiante afronte su aprendizaje mediante estas metodologías activas como de la evaluación formativa que retroalimenta dicho proceso y que les ayuda a trabajar mejor durante todo el curso. De este modo, los resultados de este trabajo vienen a aportar algo más de evidencia a la literatura sobre los efectos positivos del aula invertida en el proceso de enseñanza-aprendizaje de los estudiantes en el ámbito universitario.

\section{Referencias}

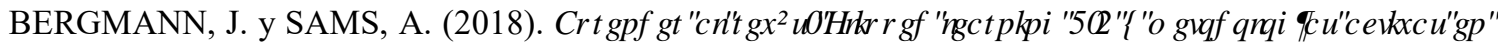
HODXФUBarcelona: Paidós Educación.

CARMINES, E.G. y ZELLER, R.A. (1979).

CHIH-YUAN, J. y WU, Y.T. (2016). "Analysis of learning achievement and teacher-student interactions

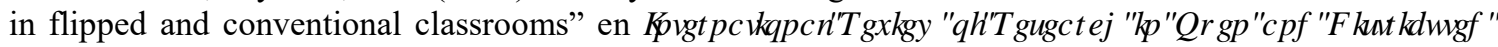
/ HDQQD, 17,1, p. 79-99.

DANKER, B. (2015). "Using Flipped Classroom Approach to Explore Deep Learning in Large Classrooms" en ,\$) 25:RXLDORIL GXFDURQ ए,1, p. 171-186.

DELGADO,V., HORTIGÜELA, D., AUSíN,V. y ABELLA,V. (2018). "El Blog como Instrumento de Mejora para la Autorregulación del Aprendizaje del Estudiante Universitario" en ( WWGRV 3 HDDJyJIFRV, 44,2, p. 171-184.

FLORES, O., DEL ARCO, I., y SILVA, P. (2016). "The flipped classroom model at the university: analysis based on professors' and students' assessment in the educational field" en ,QUAQDURQDO-RXLQDORI

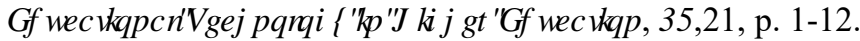

FORNELL, C. y LARCKER, D. F. (1981). "Evaluating Structural Equation Models with Unobservable Variables and Measurement Error" en -RXLQDORILO DUMQD 5 [HНDFK, 18,1, p. 39-50. 
GOODWIN, B., y MILLER, K. (2013). "Research Says. Evidence on Flipped Classrooms Is Still Coming" en 7HFQRRJ $\square 5$ IFKV HDQQD, 70,6, p.78-80.

GROSS D., PIETRI E. S., ANDERSON G., MOYANO-CAMIHORT K., y GRAHAM M. J. (2015). "Increased preclass preparation underlies student outcome improvement in the flipped classroom" en \&\% ( / IHH6FL( GXF] 14,4, 36.

HENRI, J. F. (2007). "A quantitative assessment of the reporting of Structural Equation Modeling

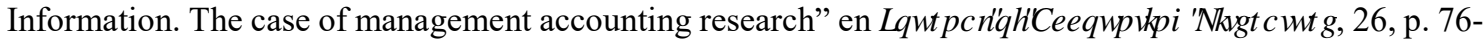
115.

HORTIGÜElA, D., PÉREZ-PUEYO, A. y GONZÁlEZ-CALVO, G. (2019). "Pero...¿A qué nos referimos realmente con la evaluación formativa y compartida? Confusiones habituales y reflexiones

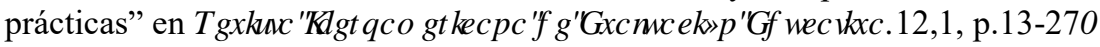

JENSEN J. L., KUMMER T. A. y GODOY P. D. (2015). "Improvements from a flipped classroom may simply be the fruits of active learning" en \&\% $\square$ IHH6FL( GXF., 14,1, 5.

MACKENZIE, S. B., PODSAKOFF, P. M., y PODSAKOFF, N. P. (2011). "Construct measurement and validation procedures in MIS and behavioral research: Integrating new and existing techniques" en $0,6 \square$ ТХDUसФ, p. 293-334.

MARTÍN, D., TOURÓN, J. (2017). "El enfoque flipped learning en estudios de magisterio: percepción de

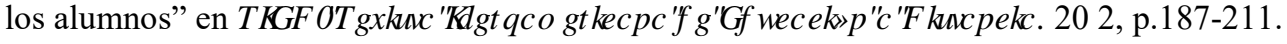

MEUSEN-BEEKMAN, K. D., JOOSTEN-TEN BRINKE, D. y BOSHUIZEN, H. P. (2015). "Developing young adolescents' self-regulation by means of formative assessment: A theoretical perspective" en \&RJHQW ( GXFDMRQ 2,1, 1071233.

MORENO-GUERRERO, A. J., JURADO DE LOS SANTOS, P., PERTEGAL-FELICES, M. L. y SOLER COSTA, R. (2020). "Bibliometric study of scientific production on the term collaborative learning in web of science" en 6XWWQDEICWW, 12,14, 5649.

MURILLO-ZAMORANO L. R., LÓPEZ-SANCHEZ J. A. y GODOY-CABALLERO A. L. (2019). "How the flipped classroom affects knowledge, skills and engagement in higher education: effects on students' satisfaction" en \&RP SXWUWD D( GXFDURQ 141.

PINAR-PÉREZ, J. M., MORALES-ARSENAL, R., FERNANDEZ-MOYA, M., CUADROS-SOLAS, P. y SALVADOR, C. (2021). "Mitigating deficiencies of generation $Z$ through new educational

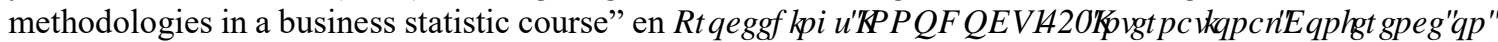
,QQRYDMRQI' RFXP HQUDURQDQCF( GXFDMRQ Valencia: Universitat Politècnica de València. 81-88.

ÖNCEL, A. F. y KARA, A. (2019). "A flipped classroom in communication systems: Student perception

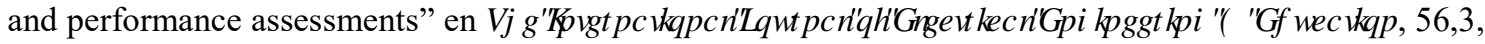
p. 208-221.

PRIETO, A., BARBARrojA, J., ÁlVAREZ, S., y CORELL, A. (2021). "Eficacia del modelo de aula invertida (flipped classroom) en la enseñanza universitaria: una síntesis de las mejores evidencias" en 5HILDOGH( GXFDFIy Q 391, p. 149-177.

PRIETO, A., y GIMÉNEZ, X. (2020A). "La enseñanza universitaria basada en la actividad del estudiante:

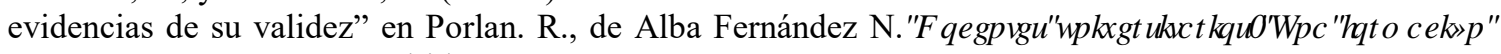
FHQWDOHQDSUS FUFD. Madrid: Morata.

PRIETO, A., DÍAZ, D., MONSERRAT, J., y BARBARROJA, J., (2020b). "La medición del impacto de las innovaciones metodológicas sobre los resultados de la docencia universitaria" en 5, ( \&6 2020.

PROBER, CHG. y HEATH, CH. (2012). "Lecture halls without lectures. A proposal for medical education" en 1 प( QJO- 0 HGH]366,18, p. 1657-1659.

RIGDON, E. E. (2012). "Rethinking Partial Least Squares Path Modeling: In Praise of Simple Methods" en / RQJ LDQJ H3 OOQQQD, 45,5-6, p. 341-358. 
ROLDÁN, J. L. y SÁNCHEZ-FRANCO, M. J. (2012). "Variance-based structural equation modeling: Guideliness for using partial least squares in information systems research" en Mora, M., Gelman, O.,

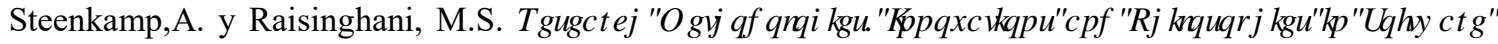
6। WAP VK( QJ IQHUQJ DDQG, QIRLP DURQ6। WHP VHershey, PA: IGI Global. p. 193-211.

SALAS, F. y VICENTE, A. (2020). "Organización de la evaluación formativa y sumativa: una experiencia

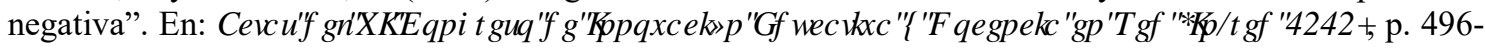
504.

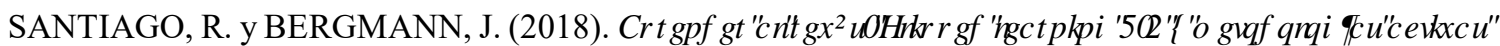
HQHODX(D) Barcelona: Paidós Educación.

STRELAN, P., OSBORN, A. y PALMER, E. (2020). "The flipped classroom: A meta-analysis of effects on student performance across disciplines and education levels" en ( GXFDMRQDO5 HMDFKD 5HIHZ, 30, 100314.

ULLMAN, J. B. (2006). "Structural Equation Modeling : Reviewing the Basics and Moving Forward" en - RXLQDORII3 HURQDOWWL WHMP HQW87, 1, p. 35-50.

WHITE, P. J., NAIDU, S., YURIEV, E., SHORT, J. L., MCLAUGHLIN, J. E. y LARSON, I. C. (2017). "Student engagement with a flipped classroom teaching design affects pharmacology examination performance in a manner dependent on question type" en \$PHUFDQ-RXLQDORI 3 KDPDFHXWFDO ( GXFDMRQ 81,9, p. 10-23.

ZHENG, L., BHAGAT, K.K., ZHEN, Y. y ZHANG, X. (2020). "The Effectiveness of the Flipped Classroom on Students' Learning Achievement and Learning Motivation: A Meta-Analysis" en Educational Technology \& Society 23,1, p. 1-15. 